\title{
Complimentary Sensitivity Function based Novel Cascade Control Structure for Automatic Generation Control
}

\author{
B S Durga Kameswari ${ }^{1}$, and Dola Gobinda Padhan $^{2 *}$ \\ ${ }^{1,2}$ Department of Electrical \& Electronics Engineering, \\ Gokaraju Rangaraju Institute of Engineering \& Technology, Hyderabad, India
}

\begin{abstract}
This paper introduces a series cascade control structure for automatic generation control. The control structure consists of two loops such as Primary loop and auxiliary loop (secondary loop). The secondary loop controller is designed using internal model control (IMC) approach. The primary loop controller is a PID controller which is tuned using desired complimentary sensitivity function. The beauty of the control structure is that it effectively nullifies the disturbances entering to the secondary loop as well as primary loop. The efficacy of the proposed controller is shown by comparing the simulation results with the existing methods in the literature.
\end{abstract}

\section{Introduction}

In electric power system design and operation Load Frequency Control (LFC) or Automatic Generation Control (AGC) is one of the most important issues for supplying sufficient and reliable electric power with good quality. The frequency of a power system is dependent on real power balance. A change in real power demand at one point of a network is reflected throughout the system by a change in frequency. Therefore, system frequency provides a useful index to indicate system generation and load imbalance. Any short-term energy imbalance will result in an instantaneous change in system frequency as the disturbance is initially offset by the kinetic energy of the rotating plant. Significant loss in the generation without an adequate system response can produce extreme frequency excursions outside the working range of the plant.

The successful operation of interconnected power systems requires the matching of total generation with total load demand and associated system losses. With time, the operating point of a power system changes, and hence, these systems may experience deviations in nominal system frequency and scheduled power exchanges to other areas, which may yield undesirable effects [1]

The primary notions of AGC for single area or multi areas power system are

- maintaining zero steady-state error for frequency deviations.

- limiting unscheduled tie line power flows between neighbouring control areas.

- better tracking for load demands and disturbances.

- maintaining less overshoot and settling time on the frequency and tie line power deviations.
There are two variables of interest, namely, frequency and tie-line power exchanges. Their variations are weighted together by a linear combination to a single variable called the ACE. The AGC problem has been augmented with the valuable research contributions from time to time, like AGC regulator designs incorporating parameter variations/uncertainties, load characteristics, excitation control, and parallel ac/dc transmission links. The microprocessor-based AGC regulator, self-tuning regulator, and adaptive AGC regulator designs have also been presented. The most recent advancement in this area is the application of concepts like neural networks, fuzzy logic, and genetic algorithms to tackle the difficulties associated with the design of AGC regulators for the power systems with nonlinear models and/or insufficient knowledge about the system required for its accurate modeling. Apart from advances in control concepts, there have been many changes during the last decade or more, such as deregulation of power industry and use of SMES, wind turbines, and PV cells as other sources of electrical energy to the system. Due to these, the control philosophies associated with AGC have changed to accommodate their dynamics and effects on overall system dynamic performance. The present study covers the critical review of a wide range of methodologies of AGC regulator designs of power systems with their salient features.

Ibraheem et al discussed a wide review of the recent philosophies in the area of AGC [1]. Also Several papers are avalable in the literature to address the concept of AGC [2-7].

In this paper, the Cascade control structure has been suggested for load frequency control for the first time. The approach is applied to single area power system non-reheated turbine only. The proposed control approach can be applied to other turbines as well as multi area power system.

The paper is organized as follows: Cascade control structure for AGC is discussed in section-2. Modeling of 
single area power system is discussed in section-3. Section-4 presents internal model control. Primary loop and secondary loop controllers design are discussed in section- 5 followed by simulation results in section- 6 . Concluding remarks are given in section-7.

\section{Cascade Control Structure for Automatic Generation Control}

The basic block diagram of series cascade control structure is shown in Fig 1. Gp1 and Gp2 are the transfer functions of primary and secondary plant models whereas $G c_{1}$ and $G c_{2}$ represent the primary and secondary loop controllers, respectively. ' $u_{2}$ ' denotes the output of the secondary controller. Output of the secondary loop is denoted by $y_{2}$ whereas y denotes the output of the primary loop. The disturbances $d_{1}$ and $d_{2}$ are entering to the input and output of the secondary plant models. Generally, Set-point response is less priority response in the case of load frequency control. The output of the primary controller serves as the setpoint for the secondary loop. It is discussed in the literature that Cascade control scheme is more effective when the secondary loop is faster than the primary loop and most of the disturbances enter the secondary loop. The governor, turbine, load and machine of the power system are collectively treated as primary process model. Secondary process is considered as first order dynamic process model.

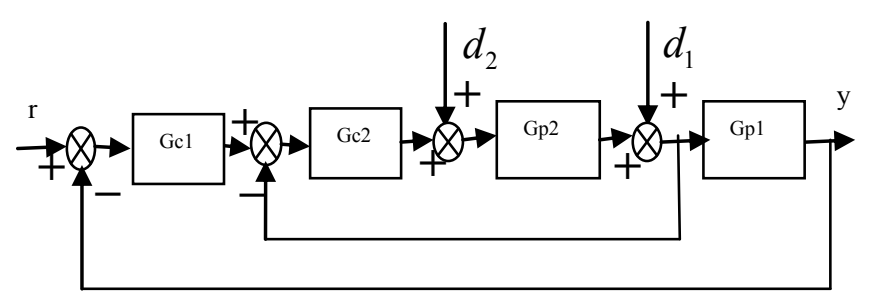

Fig. 1. Cascade control structure for AGC

\section{Modeling of a Single area power System}

Generally, power systems are highly non-linear and time-varying nature. However, for the purpose of frequency control analysis and synthesis in the face of load disturbances, a simple low-order linearized model is used. A linear model of a single-area power system is shown in Fig 2, in which a single generator is supplying power to a single-area.

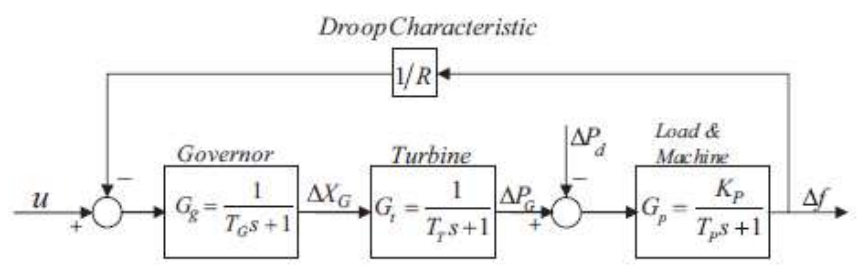

Fig. 2 Single area power system
In the present work, non-reheat turbine(NRT) is considered for LFC modeling. The plant model used for LFC without droop characteristicsis

$$
G_{p 1}=G_{g} G_{t} G_{p}
$$

where Gg, Gt and Gp are the dynamics of the governor, turbine and load \& machine, respectively.

The governor dynamics

$$
G_{g}=\frac{1}{T_{g} s+1}
$$

and

theLoad and machine dynamics,

$$
G_{p}=\frac{K_{p}}{T_{S}+1}
$$

Non-reheat turbines are first-order units.

The dynamics of the non-reheat turbine is represented as

$$
G_{t}=\frac{1}{T_{t} s+1}
$$

\section{Internal Model Control}

\subsection{Internal Model Control}

The IMC design procedure is a two-step approach that, although sub-optimal in a general (norm) sense, provides a reasonable trade of between performance and robustness. The main benefit of the IMC approach is the ability to directly specify the complementary sensitivity and sensitivity functions and, which as noted previously, directly specify the nature of the closed-loop response. The IMC control structure is shown in Fig. 3.

The IMC design procedure consists of two main steps. The first step will insure that $G_{I M C}$ is stable and causal; the second step will require $G_{I M C}$ to be proper.

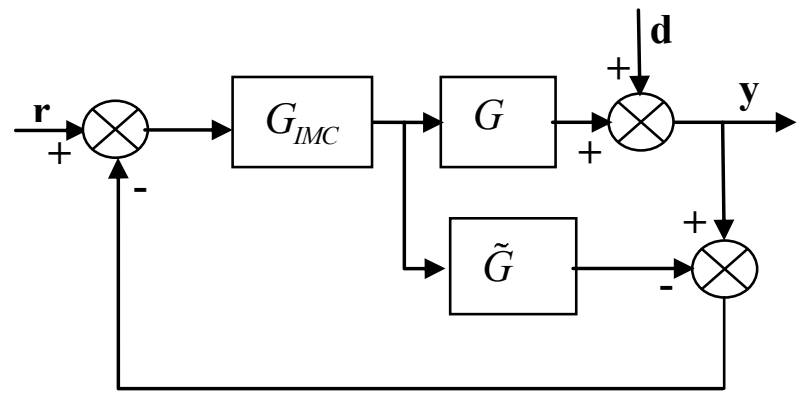

Fig. 3 IMC control structure

Decompose the plant model into two parts:

$$
\tilde{G}(\mathrm{~s})=\tilde{\mathrm{G}}_{+}(\mathrm{s}) \tilde{\mathrm{G}}_{-}(\mathrm{s})
$$

Where $\tilde{G}_{+}(\mathrm{s})$ contains all non-minimum phase terms and right half zeros.

Design a set point-tracking IMC controller as

$$
G_{I M C}(\mathrm{~s})=\widetilde{\mathrm{G}}_{-}^{-1}(\mathrm{~s}) \mathrm{F}(\mathrm{s})
$$


where $F(s)$ is a low pass filter having steady state gain of one. The filter is introduced for physical realisability of the IMC controller, $\operatorname{GIMC}(s)$. The simplest filter has the following form [9]

$$
F(\mathrm{~s})=\frac{1}{(\eta \mathrm{s}+1)^{n}}
$$

Where $\eta$ is the tuning parameter of the filter.

\section{Controller Design}

\subsection{Primary controller design}

The primary controller is considered in the following form

$$
G_{c 1}=K_{c}\left(1+\frac{1}{T_{i} s}+\frac{T_{d} s}{\varepsilon T_{d} s+1}\right)
$$

Where the derivative filter constant $\varepsilon$ is chosen as 0.1 . For load frequency deviation, the complimentary function is obtained as

$$
T=\frac{G G_{c 1}}{1+G G_{c 1}}
$$

In order to achieve the closed loop internal stability, the asymptotic stability

$$
\lim _{s \rightarrow-\frac{1}{T_{1}},-\frac{1}{T_{2}}}(1-\mathrm{T})=0
$$

should be satisfied to reject a step change in load of power system.

In the present work, the desired closed loop complimentary function is suggested as

$$
T=\frac{\left(\alpha_{2} \mathrm{~s}^{2}+\alpha_{1} \mathrm{~s}+1\right)}{(\lambda \mathrm{s}+1)^{4}} e^{-\theta_{m} s}
$$

Where $\lambda$ is a tuning parameter to achieve satisfactory closed loop performance of power system.

Using the above constraints and complimentary sensitivity function, the primary controller is obtained as

$$
G_{c 1}=\frac{\left(\mathrm{T}_{1} \mathrm{~s}+1\right)\left(\mathrm{T}_{2} \mathrm{~s}+1\right)\left(\alpha_{2} \mathrm{~s}^{2}+\alpha_{1} \mathrm{~s}+1\right)}{k\left[(\lambda \mathrm{s}+1)^{4}-\mathrm{e}^{-\theta_{m}{ }^{s}}\left(\alpha_{2} \mathrm{~s}^{2}+\alpha_{1} \mathrm{~s}+1\right)\right]}
$$

The resulting controller is not in the standard PID controller form.

So, using Laurent series $G_{c 1}$ can be expanded in the following form

$$
G_{c 1}=\frac{1}{s(\beta \mathrm{s}+1)}\left(\cdots+\gamma(0)+\gamma^{\prime}(0) \mathrm{s}+\frac{\gamma^{\prime \prime}(0) \mathrm{s}^{2}}{2 !}+\cdots\right)
$$

Now, the parameters of the primary controller can be obtained as

$$
\begin{aligned}
& K_{c}=\gamma^{\prime}(0) \\
& \frac{K_{c}}{T_{i}}=\gamma(0) \\
& \mathrm{K}_{c} T_{d}=\frac{\gamma^{\prime \prime}(0)}{2 !}
\end{aligned}
$$

\subsection{Secondary controller design}

The inner loop controller $\left(G_{c 2}\right)$ is an IMC controller and is designed based on IMC approach. The detail of the approach has been discussed in the previous section. Using the IMC approach, the secondary loop controller is obtained as

$$
G_{c 2}=\frac{\tau_{2} s+1}{k_{2}\left(\lambda_{2} s+1\right)}
$$

where $\lambda_{2}$ is the desired closed-loop time constant of the secondary controller.

\section{Simulation Results}

A non-reheated turbine studied by W Tan [8] has been considered for simulation. The model parameters of the reheat turbine are given by

$\mathrm{Kp}=120, \mathrm{Tp}=20, \mathrm{Tt}=0.3, \mathrm{Tg}=0.08$ and $\mathrm{R}=2.4$

The second order identified model without droop characteristics is

$G_{p 1}=\frac{120 e^{-0.4626 s}}{(28.4952 \mathrm{~s}+1)(0.2202 \mathrm{~s}+1)}$ and using the proposed tuning rules the controller settings are $\mathrm{Kc}=1.0326, \mathrm{Ti}=1.2116$ and $\mathrm{Td}=0.342$.

The secondary loop controller is $G_{c 2}=(\mathrm{s}+1) /(2 \mathrm{~s}+4)$.

For nominal system, the frequency deviations and the corresponding control efforts are shown in Fig-4 and Fig-5.

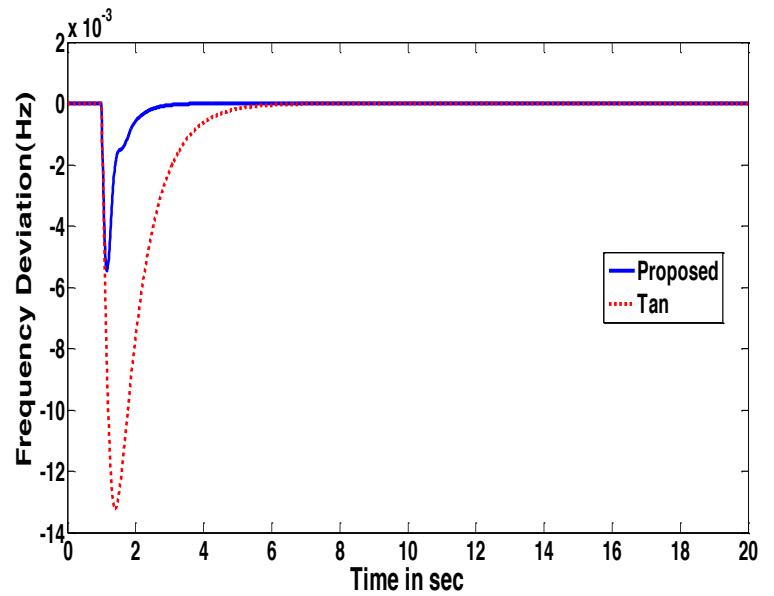


Fig. 4 Frequency deviation of the turbine without droop in nominal case

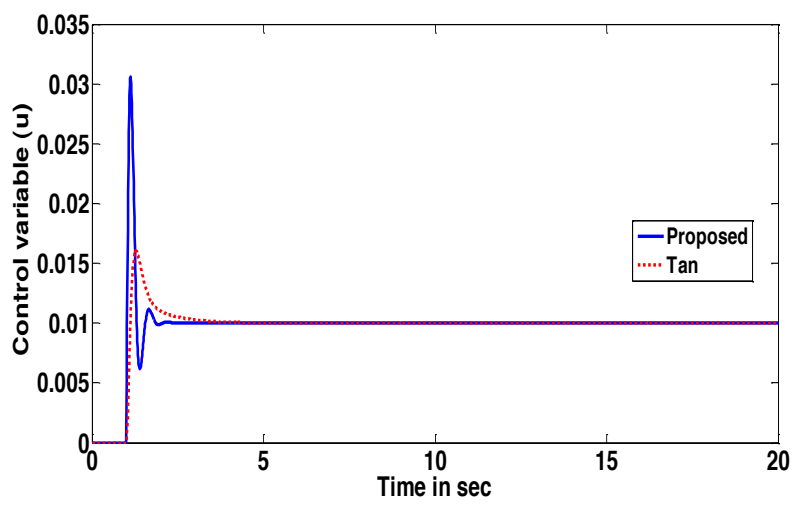

Fig. 5 Control Signal of the turbine without droop in nominal case

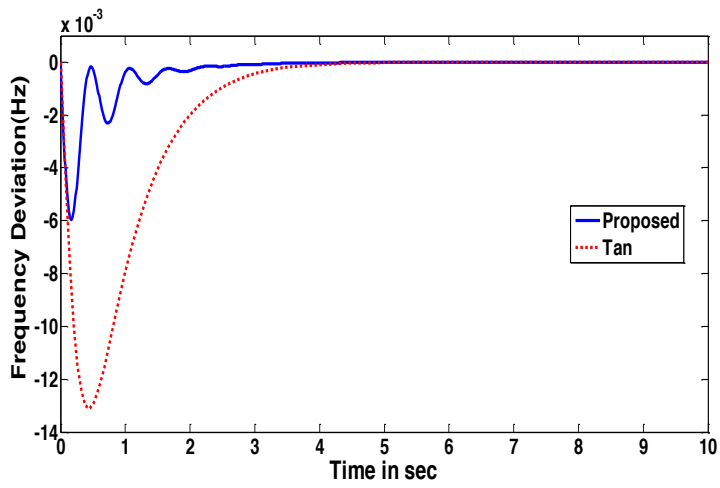

Fig. 6 Frequency deviation of the turbine without droop in perturbation case

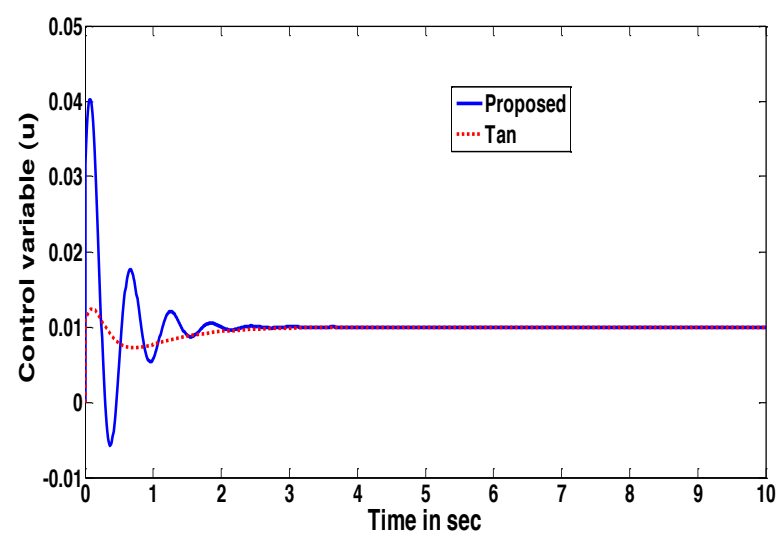

Fig. 7 Control Signal of the turbine without droop in perturbation case

The robustness of the proposed controller is tested by varying the plant parameters by $50 \%$. For perturbed system, the frequency deviation and corresponding control effort are shown in Fig-6 and Fig-7 respectively. From the simulation results, it is seen that the proposed method gives closed loop performance than Tan's approach.

\section{Conclusions}

The issues of controlling the frequency and real power deviation have been tackled by proposing a cascade control scheme for single area power system. The main merit of the cascade control structure is that it improves regulatory response. The power system parameters have been varied to demonstrate the controllers are robust and stable. The complementary sensitivity method and IMC approach have been adopted to design the primary loop and secondary loop controller respectively. It is shown in the simulation results that the proposed control scheme gives improved results when compared with existing results in the literatures. The proposed approach can be readily extended to multi-input and multi-out power system.

\section{References}

1. Ibraheem, Prabhat Kumar, Dwarka P. Kothari., Recent Philosophies of Automatic Generation Control Strategies in Power Systems, IEEE Trans. On Power Systems, 20, 1, (2005)

2. D.G. Padhan, S. Majhi, A new control scheme for PID load frequency controller of single-area and multi-area power systems, ISA Transactions, 52, 242-251, (2013)

3. Shayeghi H, Shayanfar H, Jalili A., Load frequency control strategies: a state- of-the-art survey for the researcher. Energy Conversion and Management, 50:344-53, (2009)

4. Agathoklis P, Hamza MH, Comparison of three algorithms for load frequency control, Elec. Power Sys. Research 7,165-72, (1984)

5. Park YM, Lee KY. Optimal decentralized load frequency control, Elec. Power Sys. Research, 7, 279-88, (1984).

6. Pan CT, Liaw CM. An adaptive controller for power system load frequency control. IEEE Tran. on Power Sys. 4(1), 122-8, (1989)

7. Rubaai A, Udo V. An adaptive control scheme for load-frequency control of multiarea power systems Part I. Identification and functional design. Elec. Power Sys. Research, 24,183-8 (1992).

8. Tan W., Unified tuning of PID load frequency controller for power systems via IMC. IEEE Trans. on Power systems 25(1), 341-50, (2010)

9. Ibrahim Kaya, IMC based automatic tuning method for PID controllers in a Smith predictor configuration, Computers and Chemical Engineering 28, 281-290, (2004) 